NBER WORKING PAPER SERIES

\title{
CLIMATE SENSITIVITY UNCERTAINTY: WHEN IS GOOD NEWS BAD?
}

\author{
Mark C. Freeman \\ Gernot Wagner \\ Richard J. Zeckhauser \\ Working Paper 20900 \\ http://www.nber.org/papers/w20900
}

\author{
NATIONAL BUREAU OF ECONOMIC RESEARCH \\ 1050 Massachusetts Avenue \\ Cambridge, MA 02138 \\ January 2015
}

We thank Katherine Rittenhouse for excellent research assistance and Michael Aziz, Howard Kunreuther, Chuck Mason, Ilissa Ocko, Michael Oppenheimer, Daniel Schrag, Katheline Schubert, Thomas Sterner, Martin Weitzman, Matthew Zaragoza-Watkins, and seminar participants at Duke, Harvard, the University of Gothenburg, the University of Minnesota, the 2014 American Economic Association meetings, and the 2014 World Congress of Environmental and Resource Economists for comments and discussions. All remaining errors are our own. The views expressed herein are those of the authors and do not necessarily reflect the views of the National Bureau of Economic Research. ...

NBER working papers are circulated for discussion and comment purposes. They have not been peerreviewed or been subject to the review by the NBER Board of Directors that accompanies official NBER publications.

(C) 2015 by Mark C. Freeman, Gernot Wagner, and Richard J. Zeckhauser. All rights reserved. Short sections of text, not to exceed two paragraphs, may be quoted without explicit permission provided that full credit, including $(\mathcal{C}$ notice, is given to the source. 
Climate Sensitivity Uncertainty: When is Good News Bad?

Mark C. Freeman, Gernot Wagner, and Richard J. Zeckhauser

NBER Working Paper No. 20900

January 2015

JEL No. D81,Q54

\begin{abstract}
$\underline{\text { ABSTRACT }}$
Climate change is real and dangerous. Exactly how bad it will get, however, is uncertain. Uncertainty is particularly relevant for estimates of one of the key parameters: equilibrium climate sensitivity — how eventual temperatures will react as atmospheric carbon dioxide concentrations double. Despite significant advances in climate science and increased confidence in the accuracy of the range itself, the "likely" range has been $1.5-4.5^{\circ} \mathrm{C}$ for over three decades. In 2007, the Intergovernmental Panel on Climate Change (IPCC) narrowed it to $2-4.5^{\circ} \mathrm{C}$, only to reverse its decision in 2013 , reinstating the prior range. In addition, the 2013 IPCC report removed prior mention of $3^{\circ} \mathrm{C}$ as the "best estimate."

We interpret the implications of the 2013 IPCC decision to lower the bottom of the range and excise a best estimate. Intuitively, it might seem that a lower bottom would be good news. Here we ask: When might apparently good news about climate sensitivity in fact be bad news? The lowered bottom value also implies higher uncertainty about the temperature increase, a definite bad. Under reasonable assumptions, both the lowering of the lower bound and the removal of the "best estimate" may well be bad news.

Mark C. Freeman

School of Business and Economics

Loughborough University

Leiceistershire, UK LE11 3TU

m.c.freeman@lboro.ac.uk

Gernot Wagner

Environmental Defense Fund

257 Park Avenue South

New York, NY 10010

gwagner@edf.org

Richard J. Zeckhauser

John F. Kennedy School of Government Harvard University

79 John F. Kennedy Street

Cambridge, MA 02138

and NBER

richard_zeckhauser@harvard.edu
\end{abstract}




\section{Introduction}

What if a study utilizing a new insight on equilibrium climate sensitivity-how temperatures react over time as carbon dioxide concentrations double-produced results significantly below what most climate models and scientists now assume? The natural response would be to celebrate, and to conclude that the value of stringent climate policy had diminished.

That celebration would be premature. Countervailing the good news would be the recognition that current climate science, while sound on the big picture, did not understand a fundamental climate uncertainty as well as had been thought. If there is one certainty, it is that the new data will not reveal all. Temperature increases predicted by equilibrium climate sensitivity, after all, play out over decades and centuries. Thus, the substantial change in estimates would point to even more and deeper uncertainties than previously recognized.

To put the same matter in statistical terms, our mean estimate on climate sensitivity might have shifted down, but our estimate of its standard deviation would have increased. Deeper uncertainty should magnify concerns, since marginal damages from rising temperatures increase rapidly. Quite possibly the magnified concerns from variability would outweigh any reassurance from a lowered estimate on the mean. Pindyck (2013), Weitzman (2009), Wagner and Weitzman (2015), and especially Lewandowsky et al. (2014a,b) and Pindyck (2014) discuss this tradeoff between mean and standard deviation (or variance). ${ }^{1}$

We focus here on climate sensitivity for the simple reason that it's the most iconic of climate metrics. It is also among the best-studied. Yet science has not been able to narrow its range in over three decades: double carbon dioxide concentrations and, consensus climate science tells us, expect long-run temperatures to rise by between 1.5 and $4.5^{\circ} \mathrm{C}$. That range has stood ever since J ule Charney chaired a National Academy of Sciences Ad Hoc Study Group on Carbon Dioxide and Climate in the late 1970s (Charney et al., 1979). In 1990, the Intergovernmental Panel on Climate Change (IPCC) picked up Charney's range for equilibrium climate sensitivity. ${ }^{2}$ That verdict held for a further fifteen-plus years of increasingly intense scrutiny and increased recognition of its validity until 2007, when the IPCC decided to cut off the bottom of the range, which became $2^{\circ} \mathrm{C}$. Apparent bad news: the lowest estimates for climate sensitivity seemed to be ever more out of reach.

In 2013, the IPCC took two steps with respect to the climate sensitivity parameter. First, it widened the range once again, keeping the top value of $4.5^{\circ} \mathrm{C}$ but moving the lower bound of the "likely" range back down to $1.5^{\circ} \mathrm{C}$. That step points to deep-seated uncertainties inherent in climate science and, thus, policy: thirty-five years of amazing advances in most every aspect of climate science apparently have not tightened the

\footnotetext{
${ }^{1}$ Note that this focus is different from uncertainty due to high-impact, low-probability events, often referred to as 'tail risks' or 'fat tails'. See, e.g., Weitzman (2009) and Wagner and Weitzman (2015). Tail risks magnify concerns, though they are not the focus of our analysis.

2 See Wagner and Weitzman (2015) for more on the history and the IPCC's detailed definitions.
} 
range for the eventual realization of this fundamental parameter. Indeed, they have taught us to be more cautious in defining it.

Second, the IPCC (2013) also removed its statement of a "best estimate" for climate sensitivity. No interpretation was given to this move in the report, and we don't venture to second-guess the IPCC's decision. We simply attempt to interpret the implication of removing the value itself by suggesting that any assumed climate sensitivity distribution may now have reduced 'peakedness' as a result. ${ }^{3}$ Many others have attempted to calibrate distribution functions around the IPCC's (2007) climate sensitivity pronouncements. ${ }^{4}$ The 2013 step of removing the "most likely" value might indicate that the chance of hitting close to the peak of any prior probability distribution has decreased. Once again, this additional level of uncertainty is apparent bad news: following Pindyck's (2012, 2013) calibration, decreased peakedness, other factors equal, implies greater Willingness to Pay (WTP) out of current consumption to avoid climate damages in the future.

In our subsequent analysis, we ask two sets of questions:

1. When is good news bad? Specifically, under what conditions does a lowering of the lower bound of the "likely" climate sensitivity range lead to increased WTP to avoid global warming? ${ }^{5}$

2. What should we make of the IPCC's removal of its prior "best estimate"? How do we interpret knowing about the climate sensitivity range but not where within that range we might end up? And how does this affect our WTP to avoid global warming?

We focus on the effect of increasing the uncertainty on WTP, while leaving the mean unchanged or lowering it, in section 2. Section 3 interprets the second set of questions around 'peakedness', or a lack thereof. Section 4 concludes. An extensive set of appendices presents proofs for our results, and looks for special cases with distinctive properties.

\section{When is good news bad? The mean-variance tradeoff}

A decrease in the mean climate sensitivity, ceteris paribus, is undoubtedly good news for the planet. We could expect eventual global average temperatures to rise less than previously feared. However, when that decrease in mean is due to a widening of the uncertainty range-for example, if it is due to a lowering of the lower bound-the news may not overall be good. In fact, that is what we find may be the case here.

${ }^{3}$ We interpret peakedness using kurtosis, holding the mean and standard deviation fixed. As an alternate approach, we also look at the distribution changing both its kurtosis and standard deviation to keep the IPCC's "likely" interval constant.

4 See, for example, Pindyck (2012, 2013), Weitzman (2009, 2010), and Wagner and Weitzman (2015).

${ }^{5}$ We define WTP here following Pindyck (2012, 2013), in the sense of how much it is optimal for society to pay to avoid certain degrees of average global warming in order to maximize total societal well-beingrather than in the behavioral-economic sense of the term. 
We first develop the general economic framework that underlies our analysis in subsection 2.1. Subsequently, we consider the impact of a Mean Preserving Spread (MPS) on the distribution of future temperatures in subsection 2.2. We demonstrate that the description of climate sensitivity in Assessment Report 5 (IPCC, 2013) could be seen as an MPS of the description in Assessment Report 4 (IPCC, 2007). In such a scenario, and assuming that temperature changes and economic growth are independent, then for any convex damage function and any risk averse utility function, this will lead to an increased WTP. This is despite the fact that the IPCC's 2013 position on climate sensitivity might superficially be viewed as 'good' news.

In subsection 2.3, we turn to increases in the standard deviation of the distribution of climate sensitivity. Every MPS falls into this category when the increase in variance is mean-preserving, but the converse is not true. Therefore, subsection 2.3 contains a strictly broader category of increased risk than subsection 2.2 . We demonstrate that WTP can increase even when expected climate sensitivity decreases under a broad range of conditions.

In subsection 2.4, we briefly turn our attention to the situation where there is increased uncertainty over economic growth rather than climate sensitivity.

\subsection{The framework}

Let $y_{0}$ denote current per-capita consumption levels and $y_{t}^{*}$ be what Weitzman (2010, pp. 58-59) describes as "potential consumption in the complete absence of climate change because it is defined to be what consumption would be without any global warming" at some future time $t$ (his emphasis). Then realized consumption, $y_{t}$ at time $t$ in the presence of climate change damage is given by $y_{t}=(1-D(T)) y_{t}^{*}$, where $D(T) \in$ $[0,1)$ and $D^{\prime}(T)>0$ is a multiplicative climate change damage function. We use $T$, which represents the difference between global average temperatures at time $t$ and preindustrial levels, as our proxy for climate change. It might, though, just as easily be interpreted as the rise in sea level, extreme weather events, or any other relevant climatic metric. When interpreting our results in terms of climate sensitivity, $T$ is equal to climate sensitivity due to a doubling of the level of carbon dioxide in the atmosphere.

We consider the preferences of a rational social planner in a general economic setting. $U(y, t)$ represents the planner's time-separable utility function with per-capita consumption and time as its elements. ${ }^{6}$ The amount of consumption, $p$, that the planner would be prepared to sacrifice today in order to prevent all future climate change damage at time $t$ is:

${ }^{6}$ Though we talk about a social planner acting on behalf of all individuals, the formulation could be equally well conducted in per-capita terms for a representative individual within society. This analysis excludes the 'real option' of waiting to act in the future; see, for example, Gollier (2012a, Chapter 13). As the value of real options increase with volatility, the analysis would become significantly more complex if we incorporated the ability to 'wait and see' into the model. It also ignores issues associated with the limitations of using expected utility theory in the presence of ambiguity (rather than risk), as recently discussed by Millner, Dietz and Heal (2013). 


$$
U\left(y_{0}, 0\right)-U\left(y_{0}-p, 0\right)=E\left[U\left(y_{t}^{*}, t\right)\right]-E\left[U\left(y_{t}, t\right)\right] .
$$

The left-hand side of equation (1) represents the immediate gain in utility from not spending $p$ on mitigation today, while the right-hand side is the expected gain in future utility from mitigating climate change and, thus, consuming $y_{t}^{*}$ rather than $y_{t}$. For given $y_{t}^{*}$ and $t$, we will define the value function to be $V(T)=U\left((1-D(T)) y_{t}^{*}, t\right)$.

Assume that the planner's utility function takes standard constant relative risk aversion form. No restrictions are placed on the parameter values $\rho$ and $\gamma$, which respectively represent the pure rate of time preference and the coefficient of relative risk aversion, except that the latter must be non-negative $(\gamma \geq 0)$ :

$$
U\left(y_{t}, t\right)=\left\{\begin{array}{cc}
e^{-\rho t}\left(y_{t}^{1-\gamma}-1\right) /(1-\gamma) & \gamma \neq 1 \\
e^{-\rho t} \ln \left(y_{t}\right) & \gamma=1
\end{array}\right.
$$

By substituting (2) into (1), it follows that if $g_{t}$, the per-period logarithmic growth rate in consumption in the absence of climate change damage, is defined through the relationship $g_{t}=t^{-1} \ln \left(y_{t}^{*} / y_{0}\right)$, then:

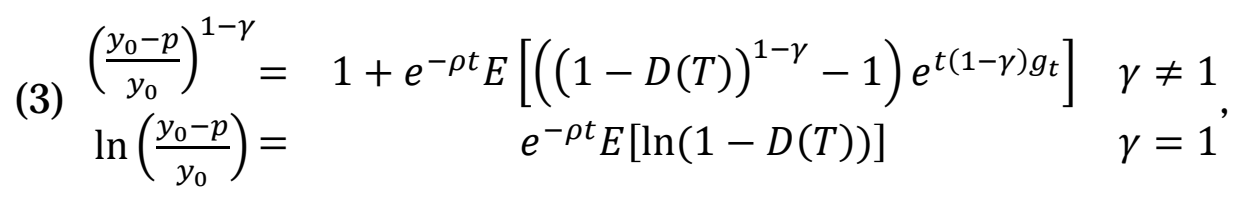

We focus on $p / y_{0}$. The value of preventative action now is directly measured by the proportion of consumption that we would willingly spend today to eliminate future climate change, all posited to be human induced. ${ }^{7}$ As the ratio increases, the implied value of policy to avoid climate change becomes stronger. This ratio is closely related to Pindyck's (2012, 2013) WTP metric, who considers what fraction of consumption society would pay to limit damage to some pre-determined level. For this analysis, for expositional ease, we set that pre-determined level equal to zero climate change. The qualitative results are the same for other values.

In general, the WTP will depend on both the change in temperature and economic growth. However, for the special case where utility is logarithmic, income and substitution effects offset each other, making $p / y_{0}$ independent of $g_{t}$ in (3).

We will denote by $f_{1}$ the probability density function that the social planner assigns to $T$ prior to the arrival of news. This density function is then amended to $f_{2}$ if the news can be interpreted as an MPS, or $f_{3}$ if the news increases variance in a way that may or may not preserve the mean.

${ }^{7}$ If there is also natural variation in climate due to nature, this could be captured by introducing an additional random factor into the analysis. 


\subsection{IPCC's lowering of the lower bound as a Mean Preserving Spread}

New information leads the social planner to update the probability density function for $T$ from $f_{1}$ to $f_{2}$. Let $x$ and $v$ be random variables drawn from $f_{1}$ and $f_{2}$ respectively. Then we say that $f_{2}$ is an MPS of $f_{1}$ if and only if we can express $v=x+z(x)$ for a random variable $z(x)$, where $E[z(x) \mid x]=0$ for all $x$ (Rothschild and Stiglitz, 1970). ${ }^{8}$

An MPS results in the variance of $f_{2}$ being greater than that of $f_{1}$, with the means of the two distributions being the same. It can also be interpreted as follows: $f_{2}$ is an MPS of $f_{1}$ if and only if $f_{1}$ second-order stochastically dominates $f_{2}$ and the two distributions have the same mean. It should be noted, though, that not every mean-preserving increase in variance can be interpreted as an MPS. (See subsection 2.3.)

We now present our main result and an immediate corollary:

Result 1. Assume $T$ and $g_{t}$ are independent. Then any MPS will increase the social planner's WTP to avoid climate change if and only if $V(T)$ is concave with respect to $T$.

Proof. See appendix A1.

Corollary 1. Any weakly risk-averse utility function and any weakly convex damage function, with at least one of these two conditions being strong, provides sufficient conditions for $V(T)$ to be concave with respect to $T$, and hence for any MPS to increase the social planner's WTP if $T$ and $g_{t}$ are independent

Proof. See appendix A1.

This is a sufficient condition for any monotonic increasing and non-convex utility function. If we restrict attention to specific utility functions, an analytic condition on the damage function that is weaker than convexity but is both necessary and sufficient can often be identified. We illustrate with the constant relative risk aversion form described in subsection 2.1.9

Corollary 2. Assume that $T$ and $g_{t}$ are independent. Any MPS in the distribution of possible future temperatures will lead a social planner who has constant relative risk aversion utility to have a higher WTP to avoid future climate change if and only if:

$$
D^{\prime \prime}(T)>-\frac{\gamma D^{\prime}(T)^{2}}{1-D(T)}
$$

${ }^{8}$ An MPS can be interpreted as follows: At each possible temperature outcome, $f_{2}$ introduces a new random gamble, $z(x)$. The distribution of $z(x)$ can vary with $x$, but the mean must be zero in all cases. Notice that this potentially includes the introduction of trivial gambles $(z(x)=0$ with probability $=1)$ for some, but not all, values of $x$, a property we will use going forward.

${ }^{9}$ Equation (A5) in the appendix provides the less analytically tractable necessary and sufficient conditions for other utility functions. 
In the case when $\gamma=1$ this result extends to the situation when $T$ and $g_{t}$ are not independent.

Proof. See appendix A1.

The proof of Corollary 2 follows from the fact that equation (4) is the necessary and sufficient condition for $V(T)$ to be concave for any constant relative risk aversion utility function. The concave curvature of $V(T)$ comes from the (weakly) concave shape of the constant relative risk aversion utility function, which more than compensates for the maximum permissible concavity of the damage function given condition (4). Given concavity for $V(T)$, from Corollary 1, greater uncertainty (an MPS) implies greater WTP to avoid climate change.

We can apply Result 1 directly to the IPCC's altered descriptions of climate sensitivity. In Assessment Report 4, IPCC (2007) stated that there is a $66 \%$ chance that climate sensitivity lies between 2 and $4.5^{\circ} \mathrm{C}$, that the most likely outcome is about $3^{\circ} \mathrm{C}$, that the probability of it being below $1.5^{\circ} \mathrm{C}$ is below $10 \%$, and that outcomes substantially higher than 4.5 degrees cannot be excluded.

For expositional ease, we illustrate our argument using a discrete distribution, $f_{1}$, for climate sensitivity. The possible outcomes are temperature increases of $\{1.40,1.75,3.00$, $4.75,6.25\}{ }^{\circ} \mathrm{C}$, with associated probabilities of $\{7 \%, 10 \%, 66 \%, 13 \%, 4 \%\} .10$

Five years later, Assessment Report 5 changed the assessment of climate sensitivity primarily by lowering the lower bound from $2^{\circ} \mathrm{C}$ to $1.5^{\circ} \mathrm{C}$, expanding the $66 \%$ likely range to 1.5 to $4.5^{\circ} \mathrm{C}$ (IPCC, 2013). Moreover, the IPCC assessed the probability of climate sensitivity less than $1^{\circ} \mathrm{C}$ at less than $5 \%$ ("extremely unlikely") and the probability that it is greater than $6^{\circ} \mathrm{C}$ at less than $10 \%$ ("very unlikely"). For expositional ease, we can once again capture this assessment with a discrete distribution, $f_{2}$, with six possible outcomes: temperature changes can equal $\{0.90,1.40,1.75,3.3214,4.75,6.25\}$ degrees, with associated probabilities $\{4 \%, 13 \%, 10 \%, 56 \%, 13 \%, 4 \%\}$.

Figure 1 plots the cumulative density functions of these two distributions.

${ }^{10}$ Contrast our discrete interpretation with that of, e.g., Weitzman (2009) or Wagner and Weitzman (2015), who use a continuous, log-normal calibration. We simply resort to a discrete function for ease of exposition. 


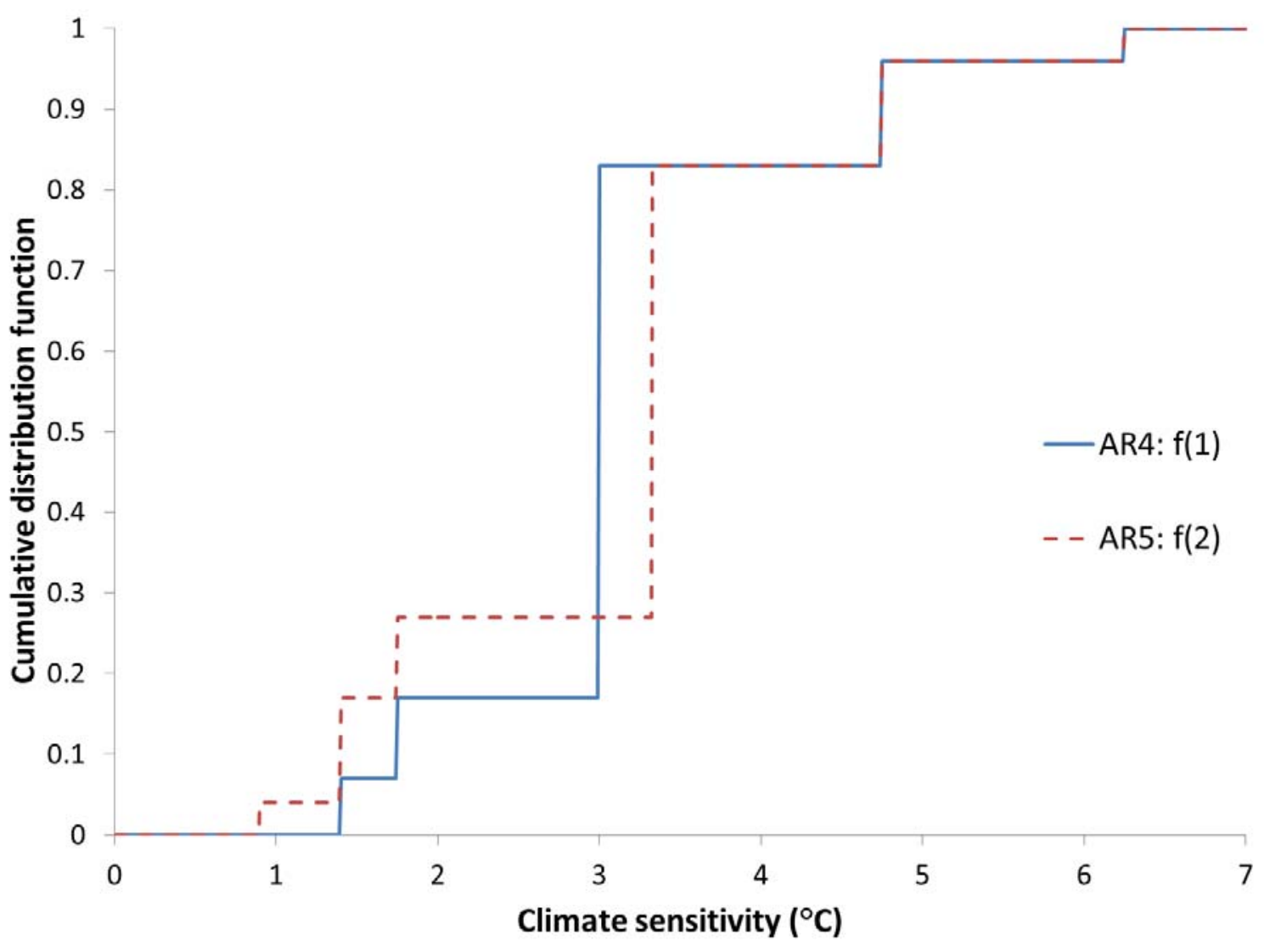

Figure 1-Cumulative distribution functions for climate sensitivity under IPCC Assessment Reports 4 (AR4) and 5 (AR5). The distributions are represented by functions $f_{1}$ and $f_{2}$, respectively.

Notice that $f_{2}$ has increased the mass in the left-hand tail. The offsetting rightward distribution of mass, so as to preserve the mean, is in the center of the distribution. There is no increase in mass in the right-hand tail.

This example is ready made for Result 1 . Define $z(x)=0$ with probability $=1$ for all values of $x$ except $x=3{ }^{\circ} \mathrm{C}$. Then set $z(3)=\{-2.1,-1.6,+0.3214\}$ with associated probabilities $\{6.06 \%, 9.09 \%, 84.85 \%\} .{ }^{11}$ Firstly, $E[z(x) \mid x]=0$ for all $x$ in this case. That's the "mean preserving" part. Second, when we add this $z(x)$ onto $f_{1}, f_{2}$ emerges. In other words, $f_{2}$ is an MPS of $f_{1}$.

Recall how $f_{1}$ is a representation of Assessment Report 4 and $f_{2}$ is a representation of Assessment Report 5's description of climate sensitivity. Result 1 then tells us that the IPCC's 2013 step to lower the lower bound in a mean-preserving way-what superficially might have appeared to be 'good' news for the planet-was indeed bad news. Positing

${ }^{11}$ One might think of the $\mathrm{z}$ gamble as scientists discovering additional uncertainties attached to the previous scenario leading to a $3^{\circ} \mathrm{C}$ temperature increase. There could also have been an MPS that added no density in either tail, and indeed was all concentrated on one or the other side of the median. 
independence of $T$ and $g_{t}$, this holds true as long as $V(T)$ is concave, which in turn holds true for any convex damage function and any weakly risk averse utility function.

\subsection{Increase in the variance of the distribution}

What if rather than an MPS, we interpreted the IPCC's step of lowering the lower bound as an increase in the standard deviation? Assume that the original distribution $f_{1}$ changed to $f_{3}$, where $\sigma_{3}>\sigma_{1}$. At this point we place no other restrictions on the two means $\left(\mu_{1}\right.$ and $\left.\mu_{3}\right)$ nor on the specific distribution of $f_{1}$ or $f_{3}$.

The situation now becomes more complex. Knowing the properties of the mean and standard deviation of the distribution of future temperatures is generally insufficient to determine the social planner's WTP. We return to this point in detail below and in the technical appendices.

\subsubsection{Logarithmic utility}

Though our initial analysis was in terms of an MPS, WTP can increase even when the expected value of climate sensitivity diminishes by a significant amount. An elegant illustration emerges if we assume a logarithmic utility and a negative quadratic exponential damage function of the type employed by Weitzman (2009) and Pindyck (2012, 2013), among others. Given these functional forms, even if IPCC (2013) reduced the expected value of climate sensitivity by a significant amount $\left(>0.05^{\circ} \mathrm{C}\right)$ when compared to IPCC (2007), the WTP could still have increased.

Result 2. Assume that the social planner has logarithmic utility and the damage function is defined as $D(T)=1-e^{-\beta T^{2}} .12$ Then moving from $f_{1}$ to $f_{3}$ increases the social planner's WTP if and only if $\sigma_{3}^{2}-\sigma_{1}^{2}>\mu_{1}^{2}-\mu_{3}^{2}$.

Proof. In the case of logarithmic utility, subsituting the damage function into the value function gives $e^{-\rho t} E[\ln (1-D(T))]=-\beta e^{-\rho t} E\left[T^{2}\right]$. This is a quadratic function with expectation determined solely by the mean and variance. Specifically, $E\left[T^{2}\right]=E^{2}[T]+$ $\operatorname{Var}[T]$, and from equation (3):

$$
\ln \left(\frac{y_{0}-p}{y_{0}}\right)=-\beta e^{-\rho t}\left(E\left[T^{2}\right]+\operatorname{Var}[T]\right) .
$$

Hence, $p$ is monotonically increasing in $E^{2}[T]+\operatorname{Var}[T]$. Therefore the social planner's WTP is greater under $f_{3}$ than $f_{1}$ if and only if $\mu_{3}^{2}+\sigma_{3}^{2}>\mu_{1}^{2}+\sigma_{1}^{2}$. QED.

Crucially, under the conditions of Result 2, any mean-preserving increase in variance

12 The damage function is convex if and only if $T<\sqrt{(2 \beta)^{-1}}$. It also satisfy the inequality of equation (4) for all $T$ when $\gamma \geq 1$, which includes log utility. For $\gamma<1$, the inequality is met if and only if $T<$ $\sqrt{(2 \beta(1-\gamma))^{-1}}$. Therefore complete convexity in the damage function is not required either for Result 2 (except in the case of risk neutrality) or Result 3 below. 
will increase WTP. For logarithmic utility and exponential quadratic damage functions this is a stronger finding than Result 1 since not all mean-preserving increases in variance are also an MPS.

Focusing on the mean climate sensitivity alone then could once again lead us astray. Consider the implications of Result 2 in the context of the discrete distribution functions for climate sensitivity given in the previous subsection. Keeping all other values the same, we can reduce the most likely value of $f_{2}$ from $3.3214^{\circ} \mathrm{C}$ to $3.25^{\circ} \mathrm{C}$ to produce $f_{3}$. The density function $f_{3}$ represents an unambiguous improvement over $f_{2}$. The mean of $f_{3}$ is less than $f_{1}: 3.08^{\circ} \mathrm{C}$ compared to $3.12^{\circ} \mathrm{C}$. Yet, as $\left(\sigma_{3}^{2}-\sigma_{1}^{2}\right)-\left(\mu_{1}^{2}-\mu_{3}^{2}\right)=0.125>0$, we would still prefer to confront $f_{1}$ rather than $f_{3}$. The WTP has gone up, even though the new distribution has a lower mean value of climate sensitivity, more mass in the lefthand tail, and the same amount of mass in the right-hand tail. That is because outcomes got worse in the middle. What at first glance might have appeared to be 'good' news is, in fact, 'bad' news. ${ }^{13}$

\subsubsection{Non-logarithmic utility}

For non-logarithmic utility-values of $\gamma \neq 1$-the impact on the social planner's WTP of moving from $f_{1}$ to $f_{3}$ is more complex. This is because no natural damage function combines with non-logarithmic utility to give a quadratic value function. We are, therefore, no longer in the comfortable world of mean-variance decision-making, where Result 2 holds. However, we conjecture that if both (i) $f_{1}$ and $f_{3}$ belong to the same major traditional families of distributions and (ii) $T$ and $g_{t}$ are independent, then a mean-preserving increase in variance will generally increase the social planner's WTP. ${ }^{14}$

While it is beyond the scope of this paper to prove this conjecture for all possible families of distributions, all possible damage functions, and all potential values of $\gamma$, we can illustrate it for a particularly relevant case. Restrict both $f_{1}$ and $f_{3}$ to be drawn from a generalized gamma distribution where the second shape parameter is set to be 2 ; $f(T): G G(\alpha, 2, \lambda)$ :

$$
f(T)=\frac{2}{\lambda \Gamma(\alpha)}\left(\frac{T}{\lambda}\right)^{2 \alpha-1} e^{-(T / \lambda)^{2}},
$$

with $\alpha, \lambda>0$ and $T \geq 0 .{ }^{15}$ Figure 2 illustrates the probability density function (6) for four different choices of parameter values. All assume that $\mu=3^{\circ} \mathrm{C}$ but vary in the value of

${ }^{13}$ This result holds if we change the most likely value of $f_{3}$ to any value higher than $3.2155^{\circ} \mathrm{C}$, while leaving all other values unchanged, when $\mu_{3}=3.06^{\circ} \mathrm{C}$.

14 If $T$ is drawn from a family of probability density functions whose members differ by location and scale only ("Two cumulative distribution functions $G 1(\cdot)$ and $G 2(\cdot)$ are said to differ only by location parameters $\alpha$ and $\beta$ if $G 1(x)=G 2(\alpha+\beta x)$ with $\beta>0$ "; Meyer 1987, p. 422), which includes normal and uniform distributions, then Result 2 will generalize to non-logarithmic utility functions. See Meyer (1987).

${ }^{15}$ This distribution is also known as a generalized normal distribution (Khodabina and Ahmadabadib, 2010). Pindyck (2012, 2013), by contrast, relies on a displaced gamma distribution for his calibration. 


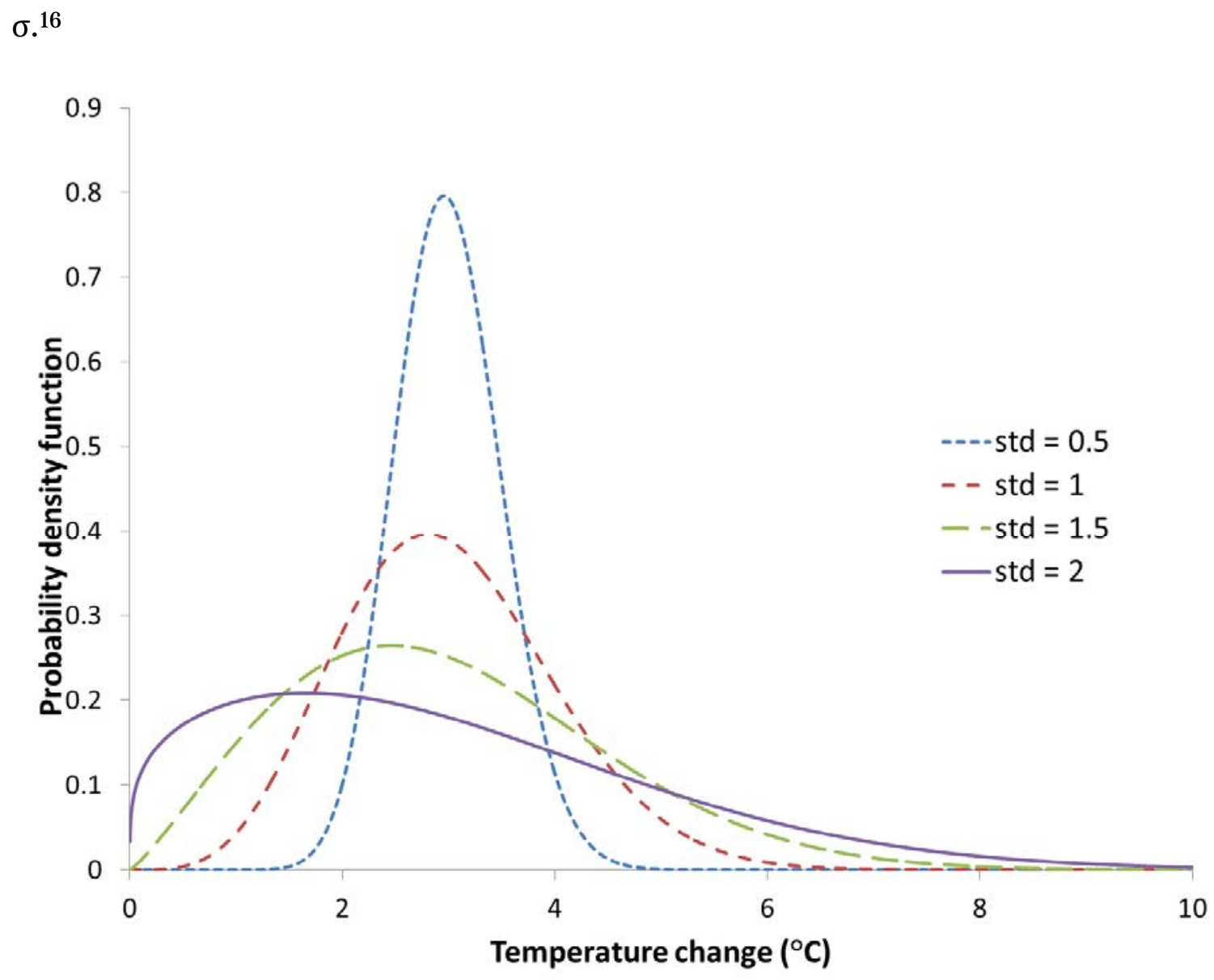

Figure 2-The probability density function of future temperature changes, which are assumed to be generalized gamma distributed with second shape parameter equal to 2: $f(T): G G(\alpha, 2, \lambda)$. The values of $\alpha$ and $\lambda$ are chosen so that the mean of $T$, $\mu=3^{\circ} \mathrm{C}$ and the standard deviation of $T, \sigma \in\left\{0.5^{\circ} \mathrm{C}, 1.0^{\circ} \mathrm{C}, 1.5^{\circ} \mathrm{C}, 2^{\circ} \mathrm{C}\right\}$.

In this parameterization, the probability density function, $g\left(T^{2}\right)$, of the square of future temperature is given by a gamma distribution $g\left(T^{2}\right)=\Gamma\left(\alpha, \lambda^{2}\right)$, where the second parameter is the scale parameter. This distribution has a well-known moment generating function.

Result 3. If $D(T)=1-e^{-\beta T^{2}}, f(T): G G(\alpha, 2, \lambda), \gamma \neq 1$ and $g_{t}$ and $T$ are independent, then any mean-preserving increase in variance raises the WTP.

Proof. We provide the full proof of Result 3 in appendix A3. The analytical derivation relies on the assumption that, for values of $\alpha$ that are likely to be relevant for describing potential future climate change damage, $(\Gamma(\alpha+0.5) / \Gamma(\alpha))^{2}$ is well approximated by $-A+\alpha$ for some positive constant $A$. The appendix also describes a series of empirical

${ }^{16}$ If we center climate change damage around $\mu=3^{\circ} \mathrm{C}$ of warming, with a standard deviation of $\sigma=1.5^{\circ} \mathrm{C}$, then this gives parameter values of $\alpha=1.0876$ and $\lambda=3.2162$. 
tests of this result which do not rely on this approximation. Under a wide range of plausible parameter values, in no case is Result 3 found to be violated.

Result 3 also implies that Result 2 extends to non-logarithmic utility for most plausible functional forms and parameter values. (See the technical appendix for counterexamples.) Figure 3 illustrates the empirical magnitude of this result. Let $p_{c}$ denote the value of $p$ if $T=\mu$ with certainty. Figure 3 presents values for $p / y_{0}$ and $p_{c} / y_{0}$ under the conditions of Result 3 and when $\rho=g_{t}=0$ for different choices of $\gamma$ (Panel A), $\sigma$ (Panel B), and $\beta$ (Panel C). ${ }^{17}$

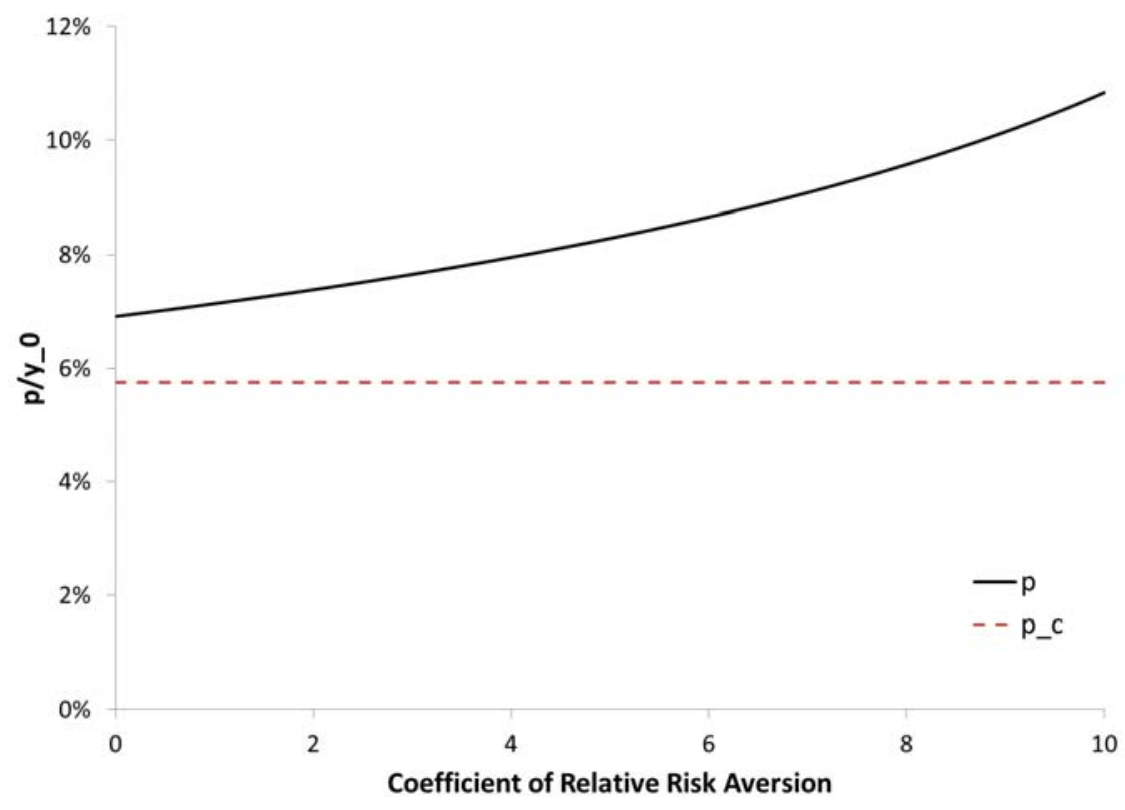

Panel A: Risk aversion

17 The baseline parameter values used in these figures are: $\mu=3^{\circ} \mathrm{C}, \sigma=1.5^{\circ} \mathrm{C}, \gamma=3$ (Panel B also includes $\gamma=0.5$ ) and $\beta=0.006585$. 


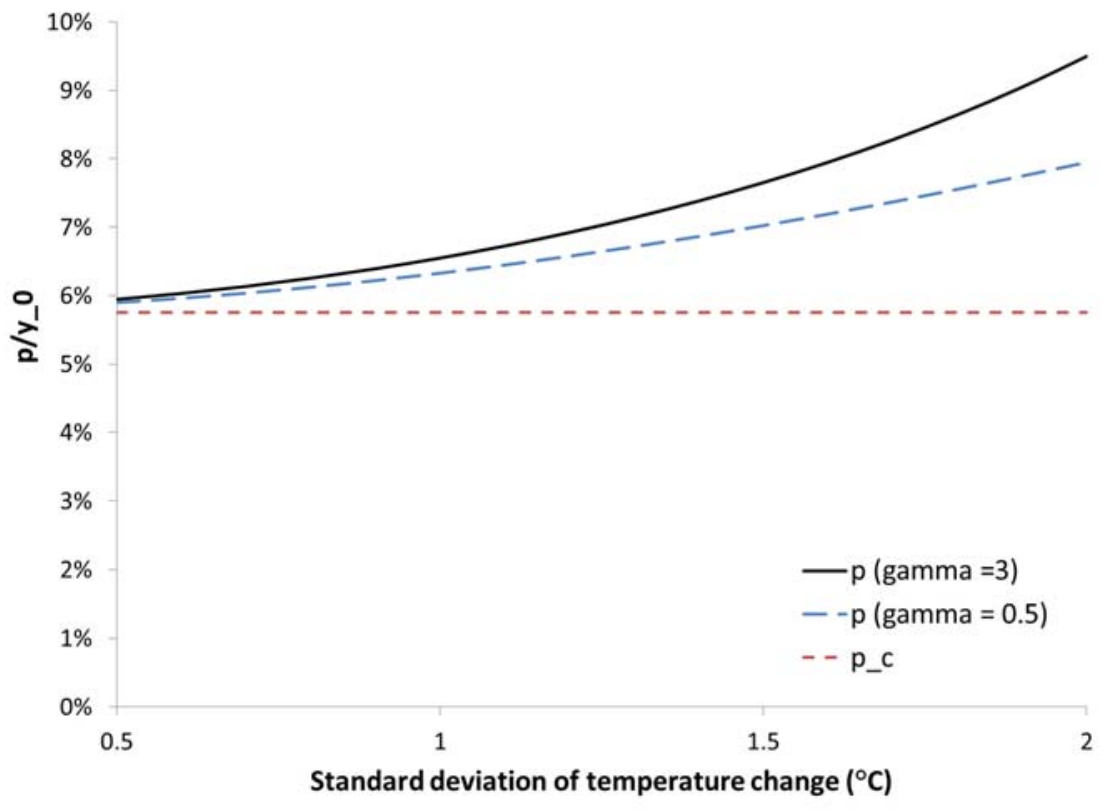

Panel B: Standard deviation of temperature

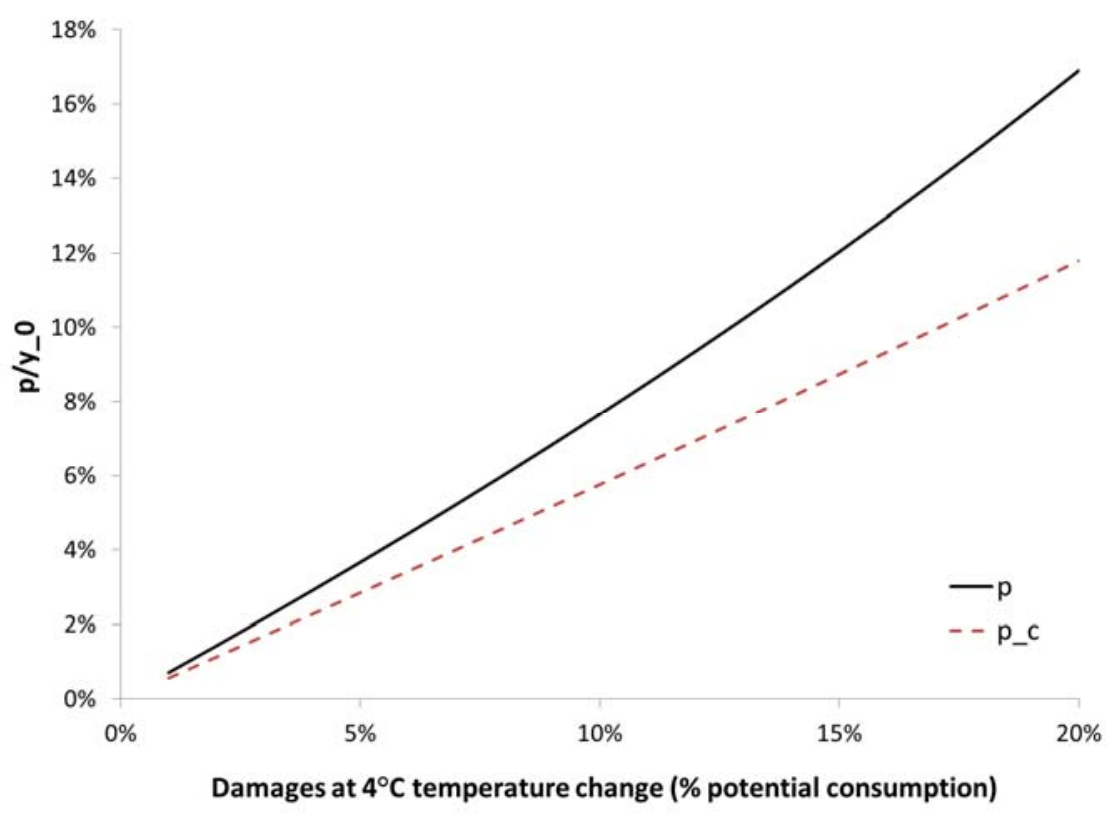

Panel C: Temperature damages

Figure 3- This figure illustrates the amount that a rational social planner would spend as a proportion of current consumption to prevent climate change both in the presence, $p$, and absence, $p_{c}$, of future temperature uncertainty. Panels A, B and C respectively vary the coefficient of relative risk aversion, $\gamma$, the standard deviation of future temperature, $\sigma$, and the extent of the damages caused by climate change $\beta$. 
Even when the social planner is risk-neutral $(\gamma=0)$, the predominantly convex damage function results in $p>p_{c}$. As $\gamma$ increases, $p / y_{0}$ rises: the more risk averse the planner is, the more the social planner is willing to pay today to tackle climate change when future temperature is uncertain. This particular illustration assumes $g_{t}=0$, although it extends to some (not all) situations when that is not the case (see the technical appendices). Moreover, as temperature uncertainty increases, so does $p / y_{0}$, consistent with Result 3. Finally, and as expected, the more that temperature change converts into actual damages (through $\beta$ ), the higher is the WTP, and the greater is the difference between $p$ and $p_{c}$.

In the technical appendices, we provide two sets of counter-examples to the general principle that WTP increases with a mean-preserving increase in the variance of the distribution that describes climate sensitivity. These are:

(i) When $f_{1}$ and $f_{3}$ are highly different probability density functions. For nonlogarithmic utility, the value function will often result in a dislike of kurtosis as well as variance. It is therefore possible to construct examples with high variance (but low kurtosis) that give the social planner a lower WTP than under an alternate distribution with lower variance (but higher kurtosis). See Technical Appendix TA1.

(ii) When the coefficient of relative risk aversion is high, and $g_{t}$ and $T$ are strongly positively correlated. In this case, the most severe tempertature damages occur when economic growth has been strong and society is richest. Because of the high coefficient of relative risk aversion, these states are discounted heavily. Therefore, the most severe temperature damages, which come when growth has been extremely positive, do not have a high present value. See Technical Appendix TA2.

While these counter-examples are of academic interest, their relevance for real world policy making is uncertain. For example, it seems unlikely that news about climate sensitivity would significantly reduce kurtosis while simultaneously raising the variance of our prevailing climate sensitivity distribution. The correlation between temperature changes and economic growth could easily go in either direction. Under one possible evolution, dramatic scientific and engineering advances in green technologies simultaenously facilitate significant economic growth and much less temperature change. In this case, the correlation between $g_{t}$ and $T$ is negative. In a much less beneficial scenario, policy makers pursue economic grouth through existing 'dirty' technologies, resulting in $g_{t}$ and $T$ being positively correlated. Given these divergent views, our base case illustrates for situations where $g_{t}$ and $T$ are uncorrelated.

\subsection{Economic growth}

So far we have focused on how changes in the distribution of climate sensitivity would affect WTP. Under a wide range of conditions, both an MPS and a potentially meanreducing increase in variance increases WTP. 
We could imagine a similar analysis with respect to uncertainty regarding economic growth. For example:

Result 4. Assume that $g_{t}$ and $T$ are independent and that $\gamma \neq 1$. Then for any damage function $D(T) \in[0,1)$ and for any probability density function describing future temperature uncertainty, WTP increases following an MPS in the distribution of future consumption growth. For logarithmic utility $(\gamma=1)$, WTP to avoid climate change is independent of the properties of $g_{t}$ even without independence from $T$.

Proof. See appendix A4.

This result draws parallels with the well-known finding around a declining social discount rate given uncertain future growth. ${ }^{18}$

\section{Removal of "best estimate" as decreased peakedness}

The previous section focused on how the increase in uncertainty that is associated with the IPCC's decision to widen the "likely" range of climate sensitivity in its Assessment Report 5 may well have increased the WTP. In this section, we turn to the other important recent change in the way it reports estimates of this parameter. The IPCC's 2007 report included a "best estimate" for climate sensitivity of $3^{\circ} \mathrm{C}$. No longer. In its 2013 report the IPCC abandoned its statement identifying a "best estimate."19

We interpret the resulting change as one that seems to be best captured by a look at the distribution's decreased 'peakedness'. To isolate the implications, we shift our analysis of climate sensitivity to a normal distribution while keeping mean and standard deviation constant. In particular, we use a probability-density function considered by Zeckhauser and Thompson (1970):

$$
f(z ; \mu, \sigma, \theta)=[2 \sigma \Gamma(1+1 / \theta)]^{-1} \exp \left\{-\left|\frac{z-\mu}{\sigma}\right|^{\theta}\right\}
$$

with $\sigma>0$ and $\theta>0$, where $\theta$ defines the distribution's kurtosis: the higher is $\theta$, the lower is its kurtosis, and vice versa. Trimming the peak in effect decreases the kurtosis of the distribution. Figure 4 shows the effects: the higher is $\theta$, the lower is the distribution's peakedness. ${ }^{20}$

18 See, for example, Arrow et al. (2013, 2014) and Cropper et al. (2014).

19 There is a mention of a "mean" climate sensitivity parameter of $3.2^{\circ} \mathrm{C}$ in Chapter 12 of the full IPCC report. However, in a break from IPCC (2007), neither the Summary for Policy Makers nor the chapter summaries themselves include a statement of the "best estimate" (IPCC 2013, and Wagner and Weitzman, 2015).

${ }^{20}$ Importantly, Zeckhauser and Thompson's (1970) $\theta$ is not equal to kurtosis. It is rather a parameter that directly affects kurtosis (what we call peakedness throughout the text), even though it operates in the opposite direction. For a normal distribution, $\theta=2$, while kurtosis $=3$. $\theta$ increases with a decrease in peakedness. The kurtosis of a uniform distribution equals 1.8, while $\theta=\infty$. 


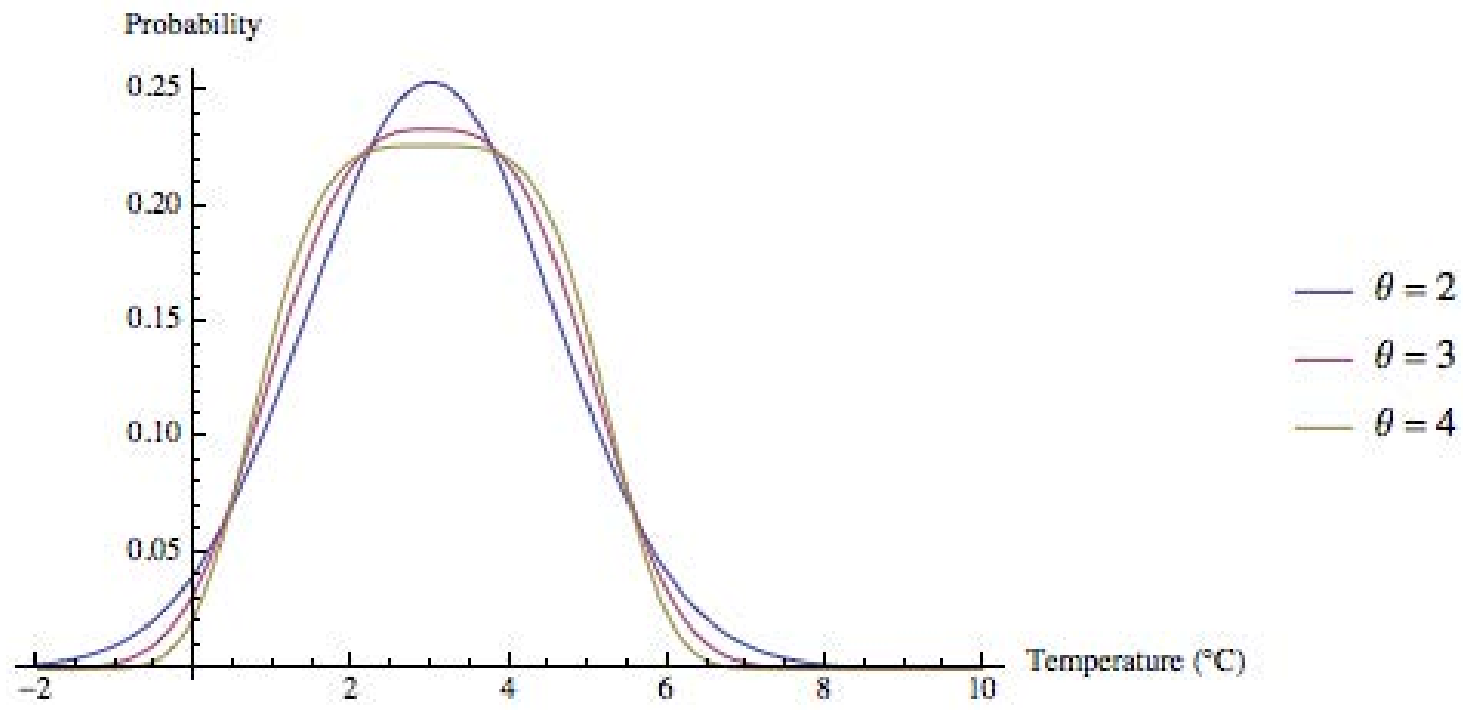

Figure 4-Climate sensitivity distribution, calibrating a standard normal distribution to the IPCC's "likely" range of 1.5 to $4.5^{\circ} \mathrm{C}$.

The assumed distribution is symmetric, and is not cut off at zero. These properties make it far from perfect to describe climate sensitivity. In fact, they tilt our results toward conservatism in the sense that we are clearly underestimating the true uncertainties involved, in particular on the upper tail of what might more accurately be captured by a skewed distribution.

Result 5. Removing the "best estimate" for climate sensitivity, a step we interpret as decreasing peakedness of the distribution, increases WTP.

Proof. We rely on Pindyck's (2012, 2013) model to analyze the implications of varying kurtosis, plugging in the Zeckhauser-Thompson distribution from (7) in lieu of Pindyck's displaced gamma distribution. The lower is peakedness (the higher is $\theta$ ), the higher is the WTP to avoid damages from climate change, in particular for a constant $1.5-4.5^{\circ} \mathrm{C}$ "likely" range (Figure 5). 


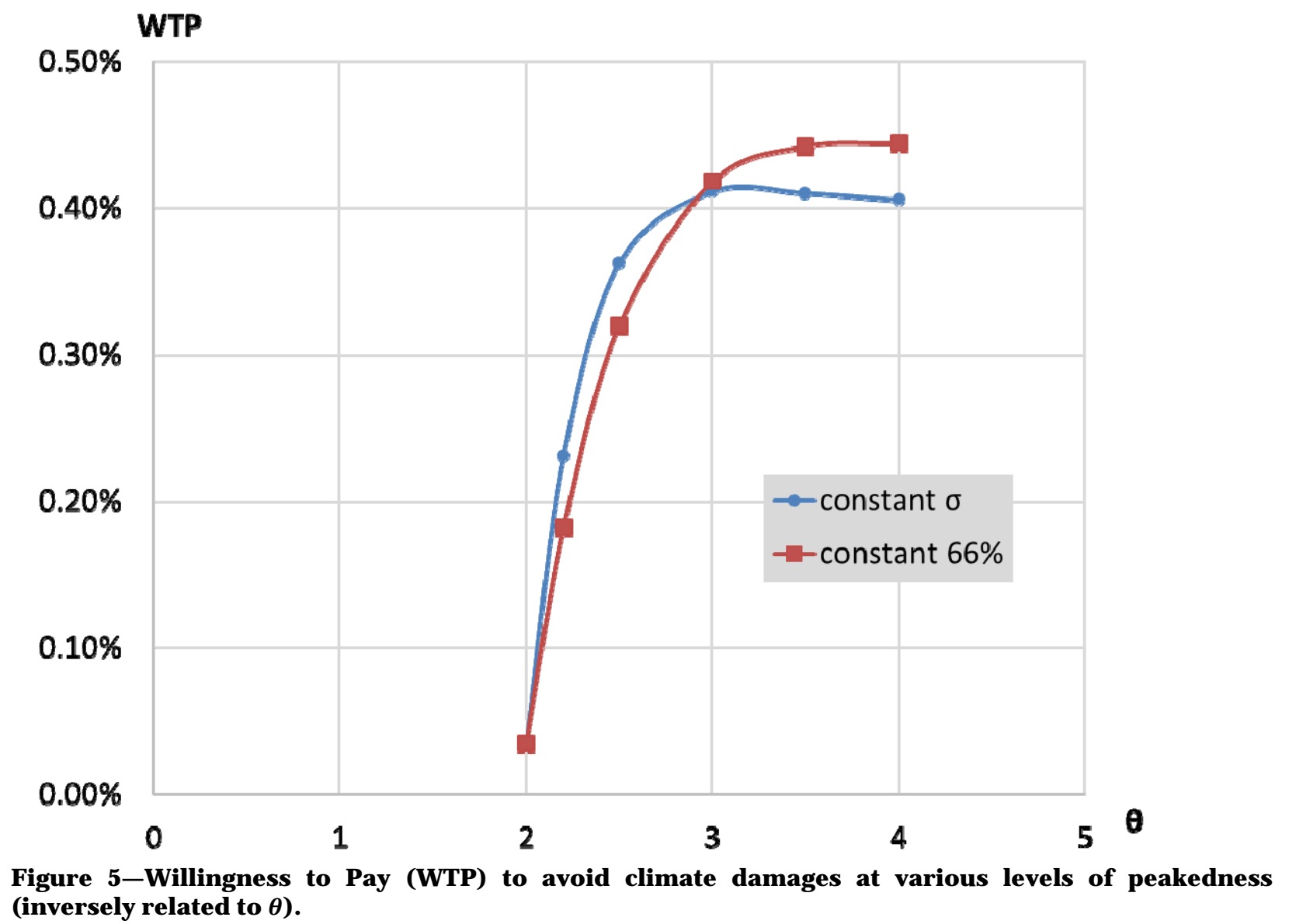
(inversely related to $\theta$ ).

"Constant $\sigma$ " in Figure 5 shows the results of varying $\theta$, while keeping everything else constant. That shows the cleanest possible trade-off of various levels of peakedness. However, it also changes the probabilities of climate sensitivity between 1.5 and $4.5^{\circ} \mathrm{C}$. In particular, increasing $\theta$ without other adjustments increases the likelihood of being between 1.5 and $4.5^{\circ} \mathrm{C}$, cutting off tails (on both ends) even further.

By adjusting the standard deviation, $\sigma$, in addition to $\theta$, we keep the IPCC's "likely" probability constant. The difference is small but important in itself. A constant "likely" probability guarantees that WTP is a strictly increasing function for reasonable values of $\theta$. Any amount of increased uncertainty within the $66 \%$ likely range-a decrease in peakedness-leads to an increased WTP to avoid climate damages.

Importantly, this result plays out entirely within the "likely" climate sensitivity range. Higher $\theta$ (lower peakedness) implies less density in the tails, which typically drive the results. ${ }^{21}$ Hence, here WTP increases with decreased peakedness despite decreased mass in the upper tail, not because of it. That decreased density in the upper tail is also the

${ }^{21}$ Weitzman (2009), Wagner and Weitzman (2015). 
reason why the WTP-line for constant standard deviation tilts downward slightly with $\theta$ above 3, and why the WTP for a constant 66\% "likely" line tapers off. ${ }^{22}$

Given that, it is important to add a warning: Figure 5 highlights the importance of relative differences across WTP levels with different levels of peakedness; the absolute WTP levels are largely irrelevant, as the uncertainty of climate sensitivity here does not play out in the all-important (fat) upper tail of the distribution. It only operates solely within the 1.5 to $4.5^{\circ} \mathrm{C}$ IPCC "likely" range.

\section{Conclusion}

Climate sensitivity - the eventual temperature outcome based on a doubling of carbon dioxide concentrations in the atmosphere-is a key parameter to determine long-run global average temperature outcomes and, thus, the costs of failing to take significant steps to mitigate future climate change. Doubling of carbon dioxide concentrations in the atmosphere is not a hypothetical. Concentrations are already up over 40 percent from preindustrial levels of around 280 parts per million (ppm) to around $400 \mathrm{ppm}$. At the current rate, pre-industrial levels will double well before the end of the century.

The title question is not a hypothetical either. While carbon dioxide emissions have picked up this past decade, leading to the relentless upward trend in concentrations in the atmosphere, global average surface warming has fallen short of past projections. ${ }^{23}$ Perhaps an even more fundamental reconsideration is the newly discovered importance of black carbon. Put together, these new facts have led IPCC (2013) to reconsider the full implications for long-term equilibrium warming and have led it to widen the "likely" range, once again, to 1.5 to $4.5^{\circ} \mathrm{C}$. In addition, the IPCC removed its prior "best estimate" of $3^{\circ} \mathrm{C}$.

Unambiguous conclusions are hard to reach in this arena where uncertainties are large. Bearing this caution in mind, our analysis strongly suggests that, ceteris paribus, the IPCC's recent widening of the "likely" range of temperature change by reducing its bottom value may well increase appropriately calibrated WTP to avoid such change in the future. The same holds for the removal of the "best estimate." Given the increasing marginal costs of global warming, greater uncertainty raises the return for taking action to curb greenhouse emissions - and the IPCC's steps reflect greater uncertainty.

22 If we did not have the result of diminished peakedness in the updated IPCC report, we might think that $\theta<2$, implying a higher peak but much more density in the tails as compared with a normal distribution. Whether this would be better or worse than the normal case would depend on both the form of the damage and utility functions.

${ }^{23}$ Economist (2013). See Nuccitelli \& Mann (2013) for a factual rejoinder. Cowtan and Way (2013) show how global average surface warming since 1997 might have been underestimated by half. Economist (2014) provides a summary. 


\section{Appendix}

\section{A1. Proof of Result 1 and Corollaries 1 and 2}

Let $x$ be a random variable describing $T$ before the introduction of an MPS and $v=x+$ $z(x)$ be a random variable describing $T$ after the introduction of an MPS with $E[z(x) \mid x]=$ 0 for all $x$. Let $p_{x}$ and $p_{v}$ respectively denote the amount the planner is willing to pay before and after the introduction of the MPS. Then, by rearranging equation (1) in the body of the text:

$$
U\left(y_{0}-p_{v}, 0\right)-U\left(y_{0}-p_{x}, 0\right)=E[V(x+z(x))]-E[V(x)] .
$$

Since the utility function is monotonic increasing, $p_{v}>p_{x}$ if and only if the right hand side of the previous offset equation is negative. By the law of iterated expectations, the right hand side can be rewritten as:

$$
E_{y_{t}^{*}, x}\left[E_{z(x)}\left[V(x+z(x)) \mid y_{t}^{*}, x\right]\right]-E[V(x)] .
$$

If $V(T)$ is concave, then by J ensen's inequality:

$$
E_{y_{t}^{*}, x}\left[E_{z(x)}\left[V(x+z(x)) \mid y_{t}^{*}, x\right]\right]<E_{y_{t}^{*}, x}\left[V\left(x+E_{z(x)}\left[z(x) \mid y_{t}^{*}, x\right]\right)\right] .
$$

From the conditions of the MPS, $E[z(x) \mid x]=0$ for all $x$. The independence of temperature and growth will also ensure that $y_{t}^{*}$ will have no conditioning information for $x$. Then $E_{z(x)}\left[z(x) \mid y_{t}^{*}, x\right]=0$ and

$$
E_{z(x)}\left[V(x+z(x)) \mid y_{t}^{*}, x\right]<V(x)
$$

This establishes that the right-hand side of equation (A1) is negative, completing the proof of Result 1.

To establish Corollary 1, notice that:

$$
V^{\prime \prime}(x)=y_{t}^{* 2} D^{\prime}(T)^{2} U^{\prime \prime}\left((1-D(x)) y_{t}^{*}, t\right)-y_{t}^{*} D^{\prime \prime}(T) U^{\prime}\left((1-D(T)) y_{t}^{*}, t\right) .
$$

As $U^{\prime \prime}(\cdot)<0, D^{\prime}(\cdot)>0$ and $D^{\prime \prime}>0$ (with potentially one of the second derivatives equalling zero), then the concavity of $V(x)$ is assured. Setting the right hand side of equation (A5) to be less than zero is the general necessary and sufficient condition for WTP to increase with an MPS for any utility function.

Turn now to Corollary 2. In the case of a constant relative risk aversion utility, when $U^{\prime}(x)=x^{-\gamma}$ and $U^{\prime \prime}(x)=-\gamma x^{-(\gamma+1)}$, equation (A5) becomes: 
(A6)

$$
V^{\prime \prime}(x)=e^{-\rho t}(1-D(T))^{-(\gamma+1)} y_{t}^{*(1-\gamma)}\left[-\gamma D^{\prime}(T)^{2}-D^{\prime \prime}(T)(1-D(T))\right] .
$$

The term outside the square bracket is strictly positive. Therefore, the term inside this bracket must be negative to make the value function concave. This is equivalent to:

$$
-\gamma D^{\prime}(T)^{2}<D^{\prime \prime}(T)(1-D(T)),
$$

and Corollary 2 follows directly. Notice that, for logarithmic utility, the independence of $T$ and $g_{t}$ is not required as $g_{t}$ does not feature in pricing equation (3) in the body of the text when $\gamma=1$.

\section{A2. Proof of Result 2}

(Given in the main text.)

\section{A3. Proof of Result 3}

We prove this result analytically under the approximation that $(\Gamma(\alpha+0.5) / \Gamma(\alpha))^{2} \approx$ $-A+\alpha$ for some positive constant $A$. We then further check this result numerically without invoking this approximation.

Analytical Proof. In the case when $f(T): G G(\alpha, 2, \lambda)$ and $D(T)=1-e^{-\beta T^{2}}$, define $W(T)=\mathrm{e}^{\beta(\gamma-1) T^{2}}$ for $\gamma \neq 1$

$$
E[W(T)]=E\left[e^{\beta(\gamma-1) T^{2}}\right]=\left(1+\beta(1-\gamma) \lambda^{2}\right)^{-\alpha},
$$

where the second equality comes from the moment generating function of the gamma distribution $g\left(T^{2}\right)=\Gamma\left(\alpha, \lambda^{2}\right)$. When $f(T): G G(\alpha, 2, \lambda)$, future temperature has a mean, $\mu$, and variance, $\sigma^{2}$, given by:

$$
\mu=\frac{\lambda \Gamma(\alpha+0.5)}{\Gamma(\alpha)}
$$

and

$$
\sigma^{2}=\lambda^{2} \alpha-\mu^{2},
$$

respectively. To proceed from here algebraically is not straightforward because the term for $\mu$ is analytically intractable. We therefore invoke the approximation of Tricomi and Erdélyi (1951), who show that, for large $\alpha$ : 


$$
\frac{\lambda \Gamma(\alpha+0.5)}{\Gamma(\alpha)}=\sqrt{\alpha}-\frac{1}{8 \sqrt{\alpha}}+O\left(|\alpha|^{-2}\right) .24
$$

Ignoring the error term, this means that:

$$
\left(\frac{\lambda \Gamma(\alpha+0.5)}{\Gamma(\alpha)}\right)^{2}=\alpha-\frac{1}{4}+\frac{1}{64 \alpha} .
$$

To simplify further, limit this to the first two terms and use the approximation:

$$
\left(\frac{\lambda \Gamma(\alpha+0.5)}{\Gamma(\alpha)}\right)^{2} \approx-A+\alpha
$$

where, from the previous offset equation, we would expect $A$ to be slightly less than 0.25 .

We estimate the value of $A$ empirically by running a linear regression of $\Gamma^{2}(\alpha+0.5) /$ $\Gamma^{2}(\alpha)$ against $\alpha$ over the interval $\alpha \in[0.01,5]$. The constant from this regression is $A=0.1759$ and the gradient is 0.9817 , which is close to 1 . The fit of the linear regression is good, with an adjusted $R^{2}=99.96 \%$. The accuracy of the approximation (A13) for $A=0.1759$ is shown in Figure A1.

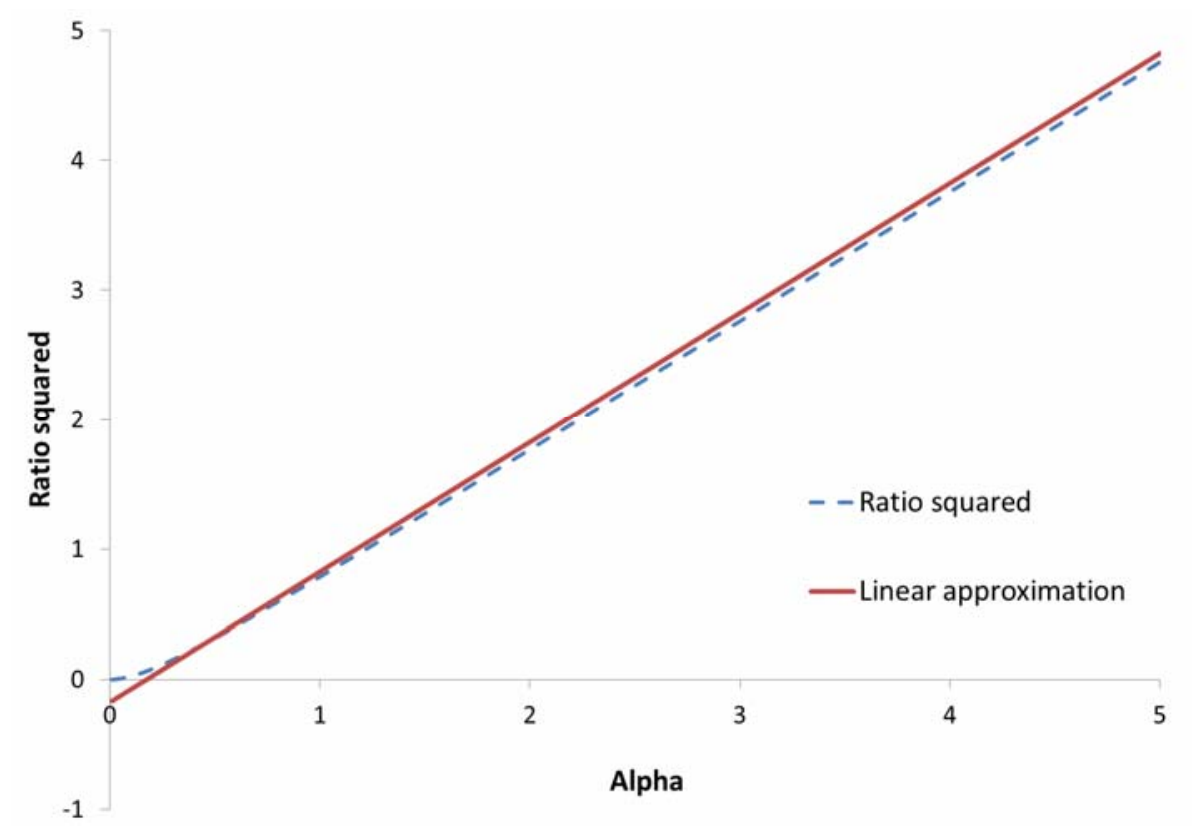

Figure A1- This shows the accuracy of the approximation $\Gamma^{2}(\alpha+0.5) / \Gamma^{2}(\alpha)=-A+\alpha$ for constant $A=0.1759$.

This linear representation performs well for values of $\alpha>0.4$. At $\alpha=0.4, \Gamma^{2}(0.4+0.5) /$ $\Gamma^{2}(0.4)=0.232$, while the approximate value $-A+0.4=0.224$.

${ }^{24}$ If $\alpha>0.5$, the accuracy of this approximation can also be considered using a double-bound given in Mortici (2010, Equation 4). 
Using this linear approximation within equation (A9) and rearranging:

$$
\alpha=A\left(\frac{\mu^{2}}{\sigma^{2}}+1\right)
$$

and

$$
\lambda=\sqrt{\frac{\sigma^{2}}{A}} .
$$

Based on $\mu=3^{\circ} \mathrm{C}$ and $\sigma=1.5^{\circ} \mathrm{C}$ respectively, this approximation gives estimates of $\alpha=0.879$ and $\lambda=3.577$, which are precisely consistent with a $G G(\alpha, 2, \lambda)$ distribution with mean of $2.93^{\circ} \mathrm{C}$ and standard deviation of $1.64^{\circ} \mathrm{C}$. Again, it is clear that the approximation works well. By substituting these expressions for $\alpha$ and $\lambda$ into equation (A8):

$$
E[W(T)]=\left(1+\beta(1-\gamma) \frac{\sigma^{2}}{A}\right)^{-A\left(\frac{\mu^{2}}{\sigma^{2}}+1\right)}
$$

under the regularity condition that $1+\beta(1-\gamma) \frac{\sigma^{2}}{A}>0$.

For notational convenience, let $X=\beta(1-\gamma) / A, Y=-A \mu^{2}$, and $Z=-A$. Then we can show that:

$$
\text { (A17) } \frac{\partial}{\partial \sigma^{2}} \mathrm{E}[\mathrm{W}(\mathrm{T})]=\mathrm{X}\left(\sigma^{2} \mathrm{X}+1\right)^{\mathrm{Z}+\frac{\mathrm{Y}}{\sigma^{2}}-1}\left(\mathrm{Z}+\frac{\mathrm{Y}}{\sigma^{2}}\right)-\frac{\mathrm{Y}}{\sigma^{4}}\left(\ln \left(\sigma^{2} \mathrm{X}+1\right)\right)\left(\sigma^{2} \mathrm{X}+1\right)^{\mathrm{Z}+\frac{\mathrm{Y}}{\sigma^{2}}} \text {, }
$$

which has the same sign as:

$$
q=X\left(Z \sigma^{4}+Y \sigma^{2}\right)-Y\left(\ln \left(1+\sigma^{2} X\right)\right)\left(\sigma^{2} X+1\right) .
$$

Now define $Q=\imath q$, where $\iota$ is an indicator variable that is equal to +1 if $\gamma>1$ and -1 if $\gamma<1$. The next step is to prove that $Q$ is strictly positive: First we can show that $Q$ is positive when $\gamma=1+\varepsilon$ for some very small value of $\varepsilon$ irrespective of the sign of $\varepsilon$. The partial derivative $\frac{d Q}{d y}$ is positive when $\gamma>1$ and negative when $\gamma<1$. This implies that, as $\gamma$ gets further away from 1 in either direction, $Q$ become more positive. Hence, $Q>0$.

Let $\gamma=1+\varepsilon$ for some very small value of $\varepsilon$ (either positive or negative). Then, to high accuracy, $\ln \left(1+\sigma^{2} X\right) \approx \sigma^{2} X$. From the previous offset equation, it follows that $Q$ has the same sign as $t\left(Z X-Y X^{2}\right)=t\left(\beta \varepsilon+\mu^{2} \beta^{2} \varepsilon^{2} / A\right)$ when $\gamma$ is close to 1 . As $\varepsilon \rightarrow 0$, so $\left(Z X-Y X^{2}\right) \rightarrow \beta \varepsilon$. Therefore, because of the presence of the indicator variable, for small $\varepsilon$ of either sign, $Q$ is positive. 
How does the sign of the partial derivative change as $\gamma$ moves further away from 1 ?

$$
\text { (A19) } \frac{\partial Q}{\partial \gamma}=\frac{\partial Q}{\partial X} \frac{\partial X}{\partial \gamma}=t\left[Z \sigma^{4}-Y \sigma^{2} \ln \left(X \sigma^{2}+1\right)\right] \frac{-\beta}{A}=t \beta \sigma^{2}\left[\sigma^{2}-\mu^{2} \ln \left(\frac{\beta(1-\gamma) \sigma^{2}}{A}+1\right)\right]
$$

has the same sign as the indicator variable if and only if:

$$
\gamma>1-\frac{A}{\beta \sigma^{2}}\left(\exp \left(\frac{\sigma^{2}}{\mu^{2}}\right)-\iota\right) .
$$

Under the baseline calibration of $A=0.1759, \beta=0.006585, \mu=3$, and $\sigma=1.5$, this places a restriction that $\gamma>-2.37$. Therefore the partial derivative is positive for all risk-averse decision makers.

This has established that, as variance increases, all else unchanged, $E[W(T)]$ increases (decreases) for $\gamma>1(\gamma<1)$. Substituting the damage function into equation (3) in the body of the text, for non-logarithmic utility and independent temperature change and growth,

$$
\left(\frac{y_{0}-p}{y_{0}}\right)^{1-\gamma}=1+e^{-\rho t} E[(W(T)-1)] E\left[e^{t(1-\gamma) g_{t}}\right]
$$

Increasing uncertainty in temperature therefore increases (decreases) the right hand side of equation (A21) for $\gamma>1(\gamma<1)$. It is easily established that the left hand side of this equation is monotonic increasing (decreasing) in $p$ for $\gamma>1(\gamma<1)$. QED.

In order to check that this result is not entirely dependent on the approximation in equation (A15), we run a range of empirical estimates of $E[V(T)]$ for $\mu \in\left[0.5^{\circ} \mathrm{C}, 6^{\circ} \mathrm{C}\right]$, $\sigma \in[0.1 \mu, 0.6 \mu] \beta \in[0.002,0.01]$, and $\gamma \in[0,7]$. At the lower bound of $\beta$, climate change damage at $4^{\circ} \mathrm{C}$ is $3.1 \%$ of potential GDP. At the upper bound, it is $14.8 \%$. For each value of $\mu$ and $\sigma$, we find consistent values of $\alpha$ and $\lambda$. We then exclude calibrations where $1+1 /\left(0.01 \lambda^{-2}\right)<9$ since the left hand side of this expression is the upper bound for $\gamma$ when $\beta$ takes its maximum value of 0.01 (see equation (A8)). We then select a range of values for $\beta$ and $\gamma$ in order to calculate $E[W(T)]$. In total, we construct 1,341,270 values of this expectation. Then, for fixed $\mu, \beta$, and $\gamma$, we examine whether $p$ increases or decreases as $\sigma^{2}$ rises. In no case is Result 3 violated.

\section{A4. Proof of Result 4}

From equation (3) in the body of the text, $g_{t}$ does not enter the pricing equation for $\gamma=1$. For non-logarithmic utility, from equation (A21), $g_{t}$ enters the pricing equation through the term $E\left[\exp \left(t(1-\gamma) g_{t}\right)\right]$ when $\gamma \neq 1$ and temperature and growth are independent. As exponential functions are convex, the proof of Result 4 is analogous to that of Result 1. 
We present two further technical appendices to demonstrate that a mean-preserving increase in the variance of future temperatures does not always raise the social planner's WTP when utility is non-logarithmic.

\section{Technical Appendix TA1}

Result A1. If $D(T)=1-\exp \left(-\beta T^{2}\right), \gamma \neq 1$ and $g_{t}$ and $T$ are independent, then a mean-preserving increase in the variance of $T$ does not necessarily imply that we should take stronger action now to prevent climate change.

Proof. This is proved by counter-example. Assume that $\rho=0$ and $g_{t}=0$ with certainty. Consider the following two discrete distributions for $T$, denoted by $f_{1}, f_{2}$. Both have three possible outcome for $T$, expressed in degrees Celsius:

$\begin{array}{lclllll} & f_{1} & & & & f_{2} & \\ \text { Outcome } & 0.720^{\circ} & 3.935^{\circ} & 8.000^{\circ} & 2.461^{\circ} & 6.586^{\circ} & 8.000^{\circ} \\ \text { Probability } & 33.07 \% & 63.79 \% & 3.15 \% & 88.31 \% & 7.65 \% & 4.04 \% \\ \mu & & 3^{\circ} & & & 3^{\circ} & \\ \sigma & & 1.75^{\circ} & & & 1.5^{\circ} & \\ \text { Skewness } & & 0.1^{\circ} & & & 2.5^{\circ} & \\ \text { Excess Kurtosis } & & 0.1^{\circ} & & & 4.5^{\circ} & \end{array}$

Both of these distributions are 'fat-tailed' in the sense of Lewandowsky et al. (2014a, Section 3.1) as "values of climate sensitivity far above the central location of the distribution are more likely than values far below". Here, both probability density functions allow for outcomes $5^{\circ} \mathrm{C}$ above the mean, but prohibit temperatures more than $2.28^{\circ}$ below the mean. The distributions are also fat-tailed in the sense that each has positive excess kurtosis. 25

Let $\beta=0.006585$, so that all possible outcomes lie on the convex part of the damage function. Let the value of $p$ associated with these temperature distributions be given by $p_{1}, p_{2}$. If the strength of the appropriate mitigative response is positively associated with greater uncertainty (as measured by the standard deviation of $T$ ), then $p_{1}>p_{2}$. However, this is not necessarily always the case, as the following table shows:

$\begin{array}{lll} & p_{1} / y_{0} & p_{2} / y_{0} \\ \gamma=0 & 0.07378 & 0.06741 \\ \gamma=0.5 & 0.07503 & 0.06933 \\ \gamma=1.00001 & 0.07636 & 0.07140 \\ \gamma=4 & 0.08645 & 0.08805\end{array}$

First, for $\gamma=1.0001$, which is almost identical to logarithmic utility, $p_{1}>p_{2}$ as expected. It is also easily verified that these values are equal to those given by Result 2 in

25 The distributions are, though, not fat-tailed in the sense of Weitzman (2009), where this term is used to characterize probability density functions with non-finite moment generating functions. 
the body of the paper (to within an approximation error reflecting the fact that $\gamma \approx 1$ ). For low values of $\gamma$, it continues to be the case that higher uncertainty strengthens the argument to mitigate now, as measured by $p$. But, for $\gamma=4$, the situation is reversed, with the higher value of $p$ being associated with the distribution with the lower standard deviation. This counterexample completes the proof.

The word "necessarily" is crucial in Result A1. As is clear from the first two rows of the last table, and is discussed in more detail in the body of the paper, it is generally the case that when $g_{t}$ and $T$ are independent and $\gamma \neq 1$, increasing the standard deviation of $T$ will increase $p$. Yet, as shown here, it is straightforward to construct counter-examples to this rule. To understand the intuition behind this result, notice that from equation (3) in the body of the paper when $\rho=0$ and $g_{t}=0$ with certainty:

$$
\left(\frac{y_{0}-p}{y_{0}}\right)^{1-\gamma}=E\left[\exp \left(\beta(\gamma-1) T^{2}\right)\right]
$$

The expectation of the exponential of a random variable is determined by all of its moments and not just the first two. ${ }^{26}$ For example, if we take a second order Taylor's series expansion of the left hand side of the previous offset equation around 1, and a second order power series expansion of the exponential function on the right hand side, for $\gamma \neq 0$, it follows that $p / y_{0}$ is approximately given by:

$$
\frac{p}{y_{0}} \approx \frac{1}{\gamma}\left(\sqrt{1+2 \beta \gamma\left(\mu^{2}+\sigma^{2}\right)+\beta^{2} \gamma(\gamma-1) E\left[T^{4}\right]}-1\right)
$$

For fixed mean and variance, increasing (decreasing) $E\left[T^{4}\right]$ raises the value of $p / y_{0}$ when $\gamma>1(\gamma<1)$. The effect of the fourth non-central moment of $T$ and higher order terms (when the power expansion is taken beyond the second term), can dominate the impact of $\sigma^{2}$, which can lead to instances where $p$ is higher for distributions with lower standard deviation.

\section{Technical Appendix TA2}

For $\gamma \neq 1$, the discussion in the main body of the paper is based on the assumption that $g_{t}$ and $T$ are independent. Relaxing this assumption necessitates a numerical approach. As before, let $D(T)=1-\exp \left(-\beta T^{2}\right)$ with $\beta=0.006585, f(T): G G(\alpha, 2, \lambda)$ with $\alpha=$ $1.0876, \lambda=3.2162$, and $\rho=0$. Further, assume that per-period logarithmic consumption growth is normally distributed with constant mean, $M$, and variance, $S^{2}$, and zero autocorrelation. Then $t g_{t} \sim N\left(t M, t S^{2}\right)$. We set $M=1.9 \%$ and $S=3.0 \%$, which are broadly consistent with long-run historical real per-capita annual consumption growth in the US.

26 There is a link here with portfolio theory and skewness/kurtosis preference in financial economics. An investor is a mean-variance decision maker if and only if she has quadratic utility. Equation (TA1) has parallels with exponential utility, where preferences are determined by moments higher than the second. 
We then run 25,000 simulations where $t g_{t}$ and $T$ are drawn from their respective distributions and with correlation between them of $\eta$. The value of $\frac{p}{y_{0}}$ is calculated numerically via equation (3) in the main body of the paper. This is then compared to three certainty equivalent values. These respectively correspond to the case when (i) temperature is non-stochastic and equals $\mu$ with certainty but consumption growth remains uncertain $\left(p_{1 c}\right)$, (ii) consumption growth is non-stochastic and equals $M$ with certainty but temperature change remains uncertain $\left(p_{2 c}\right)$, and (iii) all uncertainty is removed from both $T$ and $g_{t}\left(p_{3 c}\right)$. From equation (3) in the main body of the paper we can derive:

(TA3) $\frac{p_{k c}}{y_{0}}=$

$$
\begin{cases}1-\left(1+\left(\exp \left((\gamma-1) \beta \mu^{2}\right)-1\right) \exp \left(t(1-\gamma) M+0.5 t(1-\gamma)^{2} S^{2}\right)\right)^{1 /(1-\gamma)} & k=1 \\ 1-\left(1+\left(\left(1-\beta(\gamma-1) \lambda^{2}\right)^{-\alpha}-1\right) \exp (t(1-\gamma) M)\right)^{1 /(1-\gamma)} & k=2 \\ 1-\left(1+\left(\exp \left((\gamma-1) \beta \mu^{2}\right)-1\right) \exp (t(1-\gamma) M)\right)^{1 /(1-\gamma)} & k=3\end{cases}
$$

Figure TA1 shows the results: Panels A and B show the case when $t=50$ years, $\gamma=5$ (Panel A) and $\gamma=0.5$ (Panel B) for $\eta \in[-1,+1]$.

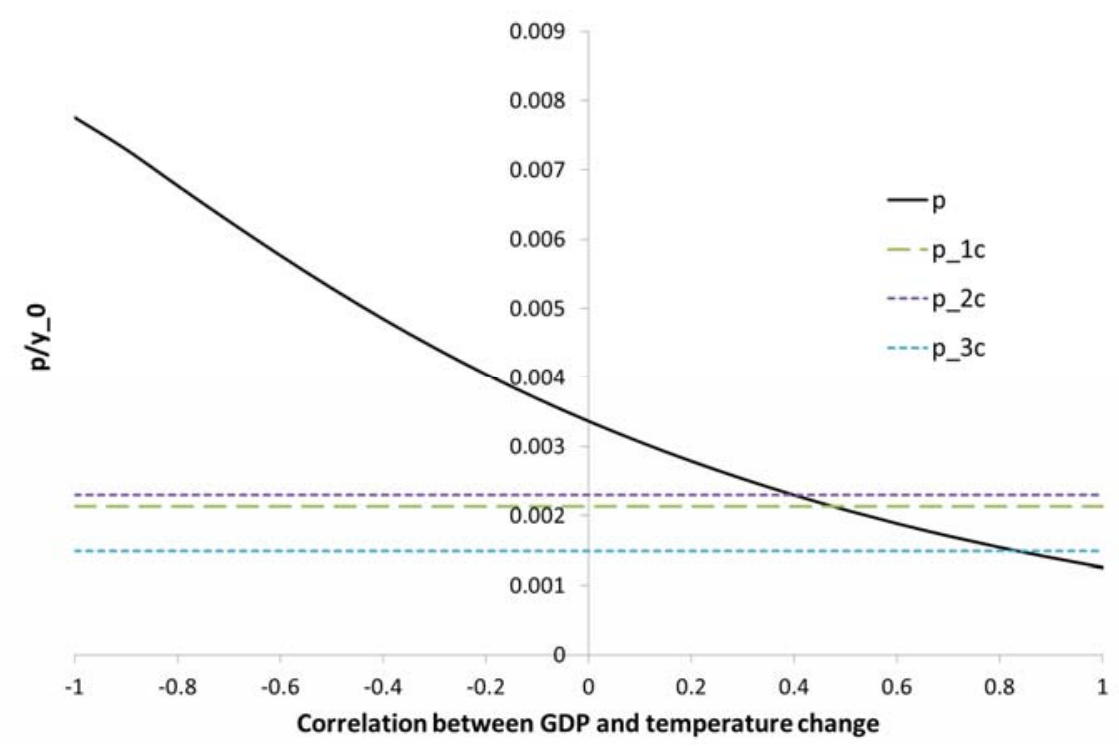

Panel A: $\gamma=5$ 


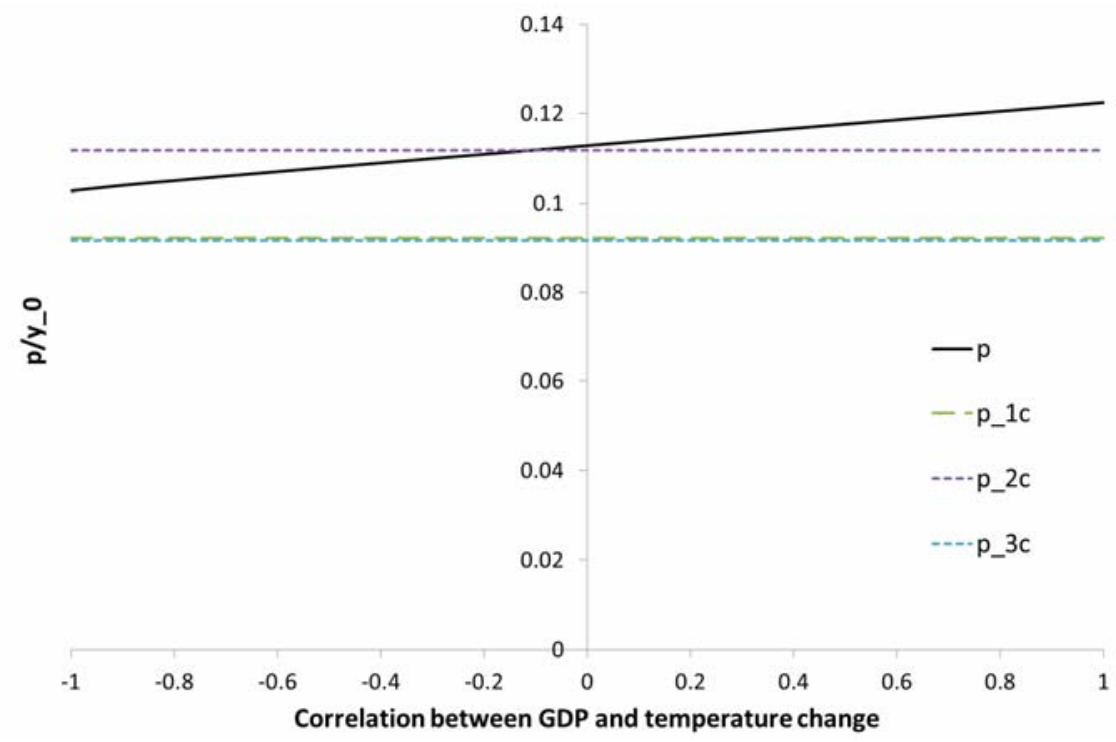

Panel B: $\gamma=0.5$

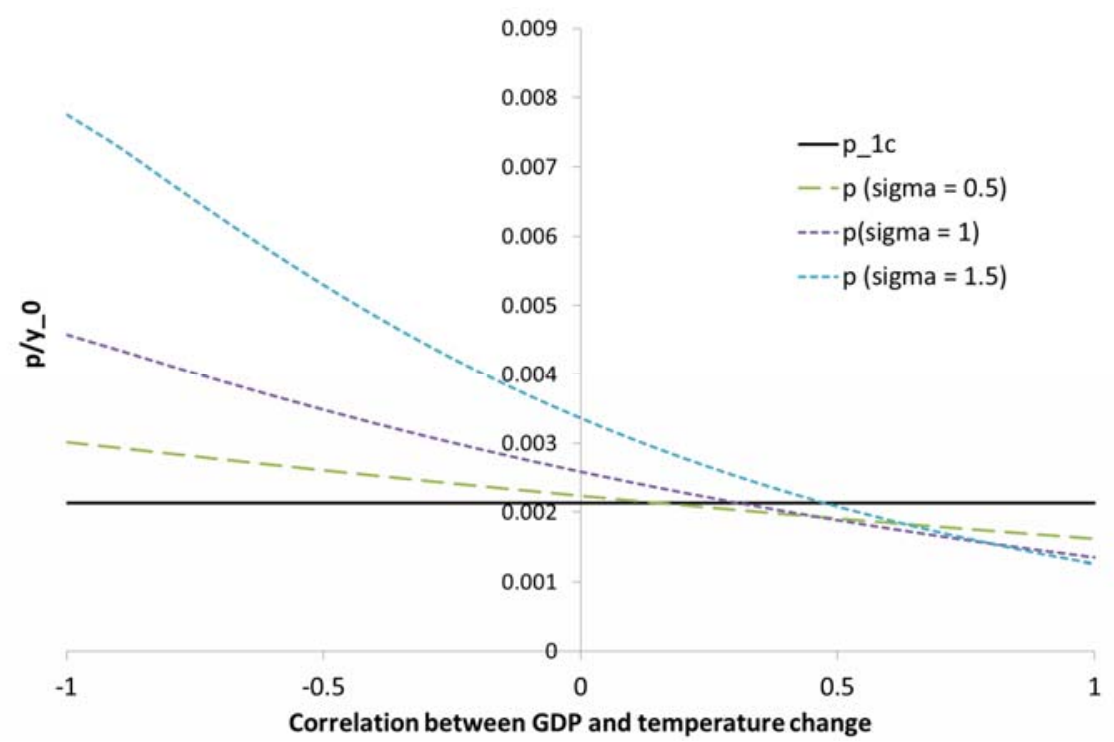

Panel C: $\gamma=5$, varying $\sigma$

Figure TA1-This figure illustrates the amount that a rational social planner would spend as a proportion of current consumption to prevent climate change, $p$. This is when there is uncertainty concerning both future temperature levels and potential consumption growth and shown for a range of different correlations between these two variables. Other lines reflect the equivalent amounts when (i) temperature change is known but consumption is stochastic $\left(p_{1 c}\right)$, (ii) consumption is known and temperature is stochastic $\left(p_{2 c}\right)$, and (iii) they are both non-stochastic $\left(p_{3 c}\right)$. Panel A (B) are respectively for $\gamma=5(\gamma=0.5)$. Panel C reflects the case for three different values of $\sigma$ when $\gamma=5$.

The most important comparison here is between $p_{c}$ and $p_{1 c}$, reflecting the presence and absence of temperature uncertainty when consumption growth is stochastic. Consistent with Result 3 in the body of the text, when $\eta=0, p>p_{1 c}$ in Panels A and B. However, for high $\gamma$ and strongly positive correlation (greater than approximately 0.4), then 
$p<p_{1 c}$, meaning that higher temperature uncertainty does not necessarily lead to a stronger economic rationale for acting now.

We can draw a number of further observations. Consistent with Result 4 in the body of the paper, $p>p_{2 c}$ when $\eta=0$, and $p_{1 c}>p_{3 c}$ for all $\eta$. When $T$ and $g_{t}$ are independent, stochastic consumption growth justifies a stronger climate response than known consumption growth with the same mean for a given distribution of $T$. Again, though, for highly positive $\eta$ and $\gamma=5$, situations arise where $p<p_{2 c}$. This is also true when $\gamma=0.5$ and the correlation is more than mildly negative. In addition, and in contrast to Panel A in Figure 3 of the body of the paper, stronger risk aversion is now associated with a weaker justification for taking substantive action now. The reason is that $\gamma$ captures not only the desire to smooth consumption across states at any given time but also reflects the social planner's desire to smooth consumption across time. In contrast to the examples in the previous section, where $g_{t}=0$ with certainty, consumption is now very likely to grow over the long term implying that future generations will be wealthier than our own. With higher $\gamma$, this reduces the incentive to spend money now to help with their problems. ${ }^{27}$

Panel $\mathrm{C}$ of TA1 shows the effects of varying $\sigma$ on $p / y_{0}$ in the case when $\gamma=5$. For negative and slightly positive values of $\eta$, greater uncertainty leads to higher values of $p$, increasing WTP. That is not the case for strongly positive values of $\eta .{ }^{28}$

\section{Bibliography}

Arrow, K., M. Cropper, C. Gollier, B. Groom, G. Heal, R. Newell, W. Nordhaus, Robert S. Pindyck, William A. Pizer, Paul R. Portney, Thomas Sterner, Richard S. J. Tol, and Martin L. Weitzman. 2013. “Determining Benefits and Costs for Future Generations.” Science 341, no. 6144: 349-350.

Arrow, Kenneth J., Maureen L. Cropper, Christian Gollier, Ben Groom, Geoffrey M. Heal, Richard G. Newell, William D. Nordhaus, Robert S. Pindyck, William A. Pizer, Paul R. Portney, Thomas Sterner, Richard S. J. Tol, and Martin L. Weitzman. 2014. "Should Governments Use a Declining Discount Rate in Project Analysis?” Rev Environ Econ Policy 8: 145-163.

Charney, J ule G., Akio Arakawa, D. J ames Baker, Bert Bolin, Robert E. Dickinson, Richard M. Goody, Cecil E. Leith, Henry M. Stommel, and Carl I. Wunsch. 1979. "Carbon dioxide and climate: a scientific assessment." National Academy of Sciences.

Cowtan, Kevin, and Robert G. Way. 2013. "Coverage bias in the HadCRUT4 temperature series and its impact on recent temperature trends." Quarterly J ournal of the Royal Meteorological Society.

Cropper ML, Freeman MC, Groom B, Pizer WA. 2014. “Declining discount rates.” American Economic Review 104:538- 543.

27 The use of an Epstein-Zin-Weil utility function would enable social planners to separate their desire to smooth consumption across time from their desire to smooth consumption across states at any given point in time. SeeJ ensen and Traeger (2014) as well as Daniel, Litterman, and Wagner (2015).

28 This result is reminiscent of that in Gollier (2012b), who shows that the term structure of discount rates is highly dependent on the 'climate beta' of the cash flow, which reflects the correlation between growth and damages. 
Daniel, Kent, Robert B. Litterman and Gernot Wagner. 2015. "Applying Asset Pricing Theory to Calibrate the Price of Climate Risk," Mimeo.

The Economist. 2013. "Climate science: a sensitive matter." March 30th.

The Economist. 2014. "Global Warming: Who Pressed the Pause Button." March 8"

Gollier Christian.2012a. Pricing the planet's future: The economics of discounting in an uncertain world. Princeton University Press, Princeton.

Gollier Christian. 2012b. "Evaluation of long-dated investments under uncertain growth trend, volatility and catastrophes." CESifo Working Paper: Industrial Organisation, No. 4052.

Intergovernmental Panel on Climate Change (IPCC). 2007. Fourth Assessment Report, Working Group I.

Intergovernmental Panel on Climate Change (IPCC). 2013. Fifth Assessment Report, Working Group I.

J ensen S, Traeger CP. 2014. “Optimal climate change mitigation under long-term growth uncertainty: Stochastic integrated assessment and analytic findings." European Econonomic Review 69 104- 125.

Khodabina M, Ahmadabadib A. 2010. "Some properties of generalized gamma distribution." Mathematical Sciences 4:9- 28.

Lewandowsky S, Risbey J S, Smithson M, Newell BR. 2014a. "Scientific uncertainty and climate change: Part I. Uncertainty and unabated emissions." Climatic Change, doi: 10.1007/s10584-014-1082-7.

Lewandowsky S, Risbey J S, Smithson M, Newell BR. 2014b. "Scientific uncertainty and climate change: Part II. Uncertainty and mitigation." Climatic Change, doi: 10.1007/ s10584-0141083-6.

Meyer, J . 1987. “Two-moment decision models and expected utility maximization.” American Economic Review 77: 421-430.

Millner A, Dietz S, Heal G. 2013. "Scientific ambiguity and climate policy.” Environmental and Resource Economics 55: 21- 46.

Mortici C. 2010. "New approximation formulas for evaluating the ratio of gamma functions." Mathematical and Computational Modelling 52:425- 433.

Nuccitelli, Dana and Michael E. Mann. 2013. " How The Economist got it wrong."Australian Broadcasting Corporation Environment, 12 April.

Office of Management and Budget (OMB). 1992. "Circular No. A-94 Revised," October 29.

Pindyck, Robert S. 2012. “Uncertain outcomes and climate change policy.” J ournal of Environmental Economics and Management 63, no. 3: 289-303.

Pindyck, Robert S. 2013. “The Climate Policy Dilemma." Review of Environmental Economics and Policy vol. 7(2):219-237.

Pindyck, Robert S. 2014. "Risk and return in the design of environmental policy." J ournal of the Association of Environmental and Resource Economists.

Rothschild, Michael, and J oseph E. Stiglitz. "Increasing risk: I. A definition." J ournal of Economic theory 2, no. 3 (1970): 225-243.

Tricomi FG, Erdélyi A. 1951. "The asymptotic expansion of a ratio of gamma functions.” Pacific J ournal of Mathematics 1: 133-142.

Wagner, Gernot and Martin L. Weitzman. 2015. Climate Shock: the Economic Consequences of a Hotter Planet. Princeton University Press.

Weitzman, Martin L. 2009. "On Modeling and Interpreting the Economics of Catastrophic Climate Change." Review of Economics and Statistics 91(1): 1- 19.

Weitzman, Martin L. 2010. "What Is The "Damages Function" For Global Warming-And What Difference Might It Make?." Climate Change Economics 1, no. 01: 57-69.

Zeckhauser, Richard, and Mark Thompson. 1970. "Linear regression with non-normal error terms." The Review of Economics and Statistics 52, no. 3: 280-286. 